\title{
DNA conformation dictates strength and flocculation in DNA-microtubule composites
}

Karthik R. Peddireddy ${ }^{1}$, Davide Michieletto ${ }^{2,3}$, Gina Aguirre ${ }^{1}$, Jonathan Garamella ${ }^{1}$, Pawan Khanal $^{1}$, and Rae M. Robertson-Anderson ${ }^{1, *}$

${ }^{1}$ Department of Physics and Biophysics, University of San Diego, 5998 Alcala Park, San Diego, CA 92110 , United States

${ }^{2}$ School of Physics and Astronomy, University of Edinburgh, Peter Guthrie Tait Road, Edinburgh, EH9 3FD, UK, ${ }^{3}$ MRC Human Genetics Unit, Institute of Genetics and Molecular Medicine University of Edinburgh, Edinburgh EH4 2XU, UK

*Corresponding author: randerson@ sandiego.edu

\section{SUPPORTING INFORMATION}

\section{Section S1. Expanded Experimental Section}

Figure S1. Measured forces in response to strain of rate $\dot{\gamma}=9.4 \mathrm{~s}^{-1}$ for DNA-MT composites.

Figure S2. Nonlinear force responses of DNA-MT composites for different strain rates.

Figure S3. Nonlinear force relaxations of DNA-MT composites following strains of different rates.

Figure S4. Average spatial image autocorrelation curves of DNA-MT composites

Section 2. Theory for phase behavior of a composite of stiff and flexible polymers

Figure S5. Results of Scaled Particle Theory using the correlation blob size $\zeta$ rather than $R_{G}$ as the principal DNA lengthscale $\sigma$.

\section{Section S1. Expanded Experimental Section}

DNA: Double-stranded 115 kilobasepair DNA is prepared by replication of cloned bacterial artificial chromosomes (BACs) in E. Coli, followed by extraction, purification, and concentration using customdesigned protocols described elsewhere ${ }^{1,2}$. Supercoiled BAC DNA constructs are enzymatically treated with MluI or topoisomerase I (New England Biolabs) to convert the supercoiled constructs to linear (L) or ring (R) topology, respectively. The corresponding radii of gyration for the linear and ring topologies are $R_{G, L} \simeq 0.88 \mu \mathrm{m}$ and $R_{G, R} \simeq 0.52 \mu \mathrm{m}$. Both linear and ring DNA stock solutions are suspended in TE10 buffer (10 mM Tris- $\mathrm{HCl}(\mathrm{pH} 8), 1 \mathrm{mM}$ EDTA, $10 \mathrm{mM} \mathrm{NaCl}$ ) at concentrations (c) of $1.9 \mathrm{mg} / \mathrm{ml}$ and 1.1 $\mathrm{mg} / \mathrm{ml}$, respectively. The ring DNA solution is composed of $\sim 90 \%$ ring DNA and $10 \%$ linear chains due to shearing that occurs during the extraction and purification process. The effect of linear chain contaminants on ring DNA rheology, widely discussed elsewhere, is to extend the entanglement regime to lower frequencies and to slow relaxation timescales ${ }^{3,4}$. DNA concentration for experiments is fixed at $c=0.65$ $\mathrm{mg} / \mathrm{ml}$, which corresponds to $\sim 2.5 c_{e}$ where $c_{e}$ is the critical entanglement concentration $\left(\simeq 6 c_{L} *\right.$ for DNA, where $c_{L}{ }^{*}=(3 / 4 \pi) M / N_{A} R_{G, L}{ }^{3} \simeq 0.043 \mathrm{mg} / \mathrm{ml}$ is the polymer coil overlap concentration for linear DNA $\left.{ }^{5}\right)$. The corresponding overlap concentration for ring DNA is $c_{R}{ }^{*} \simeq 0.17 \mathrm{mg} / \mathrm{ml}$. Because the polymers are overlapping, a key lengthscale for the DNA solution is the correlation blob size, $\zeta=R_{G}\left(c / c^{*}\right)^{-v /(3 v-1)}$ 
where $v \simeq 0.588^{6,7}$, understood as the length along the polymer that behaves as an independent coil ${ }^{7}$. The blob sizes for our ring and linear DNA solutions are $\zeta_{R} \cong 185 \mathrm{~nm}$ and $\zeta_{L} \cong 102 \mathrm{~nm}$.

Microtubules (MT): Porcine brain dark tubulin (T240) and rhodamine-labeled tubulin (TL590M) are obtained from Cytoskeleton. $45.5 \mu \mathrm{M}$ stock solutions of tubulin dimers, containing 9:1 dark:labeled tubulin dimers in PEM100 buffer (100 mM PIPES (pH 6.8), $2 \mathrm{mM} \mathrm{MgCl}_{2}, 2 \mathrm{mM}$ EGTA) are flash-frozen in liquid nitrogen and stored at $-80^{\circ} \mathrm{C}$. We include rhodamine-labeled tubulin to visualize the microtubule network in each condition. The labeled dimers are quality tested by the manufacturer to ensure their polymerization efficiency is comparable to unlabeled tubulin under identical conditions ${ }^{8}$. Further, we find no evidence that addition of the labeled dimers has a discernable impact on polymerization or network formation ${ }^{9}$. To form

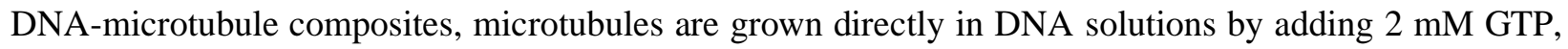
to enable polymerization, and $10 \mu \mathrm{M}$ Taxol to stabilize polymerized microtubules.

Microtubules are hollow rods with diameter $D \simeq 25 \mathrm{~nm}$, formed from $\sim 8 \mathrm{~nm}$ tubulin dimers, with 13 tubulin dimers per ring ${ }^{10}$. In the absence of DNA, the tubulin concentrations used in experiments $\left(c_{T}=2,3.5,5\right.$ and $7.5 \mu \mathrm{M}$ ) result in entangled networks with mesh sizes of $\xi_{M T}=0.89 / c_{T}{ }^{1 / 2} \simeq 1.9,1.4,1.2$, and $0.98 \mu \mathrm{m}$, respectively, where $c_{T}$ is in units of $\mathrm{mg} / \mathrm{ml}^{11}$. The distribution of lengths of the microtubules depends on the composite composition (as shown in Fig 4) but, based on images and previous work ${ }^{9,11-13}$, we estimate a distribution of lengths $L$ of $\sim 5$ to $15 \mu \mathrm{m}$. Using these estimated minimum and maximum lengths, along with the overlap concentration expression for a solution of rigid rods, $c_{R} *=\left(2^{3 / 2}\right) M /\left(N_{A} L^{3}\right)$, we compute $c_{R} *$ values of $\sim 23$ to $610 \mathrm{nM}^{14}$, well below the concentrations examined in experiments.

Sample preparation: DNA and tubulin are mixed slowly and thoroughly using wide-bore pipette tips, to prevent shearing of DNA, then introduced into sample chambers through capillary action. Sample chambers $(2 \mathrm{~cm} \times 0.3 \mathrm{~cm} \times 0.01 \mathrm{~cm})$ are made with a microscope glass slide and coverslip separated by two layers of double-sided tape and hermetically sealed with epoxy. To polymerize tubulin dimers and form DNAmicrotubule samples, sample chambers are incubated at $37^{\circ} \mathrm{C}$ for two hours, resulting in repeatable and reliable polymerization of tubulin in the DNA solutions. As an alternative method to forming composites, we also polymerized tubulin into microtubules prior to adding to DNA solutions and loading into the sample chamber. However, this method resulted in random flow alignment of microtubules that occurred when introducing the DNA-MT mixtures into the sample chambers, so the data is not shown here.

Microrheology: For microrheology experiments, a trace amount of polystyrene beads (Polysciences, Inc.) of radius $R=2.25 \mu \mathrm{m}$ are added to the DNA-tubulin mixtures prior to tubulin polymerization. Beads are coated with Alexa-488 BSA to prevent DNA adsorption and for fluorescence visualization. $0.1 \mathrm{wt} \%$ Tween 20 is added to reduce DNA and bead adsorption to the sample chamber walls. An oxygen scavenging system (45 $\mu \mathrm{g} / \mathrm{mL}$ glucose, $43 \mu \mathrm{g} / \mathrm{mL}$ glucose oxidase, $7 \mu \mathrm{g} / \mathrm{mL}$ catalase, and $5 \mu \mathrm{g} / \mathrm{mL} \beta$-mercaptoethanol) is added to inhibit photobleaching.

We use optical tweezers microrheology to determine linear and nonlinear rheological properties of the DNA-MT composites (Figure 1). Details of the experimental procedures and data analysis, briefly summarized below, have been described in detail in refs ${ }^{15,16}$. The optical trap, built around an Olympus IX71 epifluorescence microscope, is formed from a $1064 \mathrm{~nm} \mathrm{Nd:YAG} \mathrm{fiber} \mathrm{laser} \mathrm{(Manlight)} \mathrm{focused} \mathrm{with}$ a 60x 1.4 NA objective (Olympus). Forces exerted by the DNA-MT composites on the trapped beads are determined by recording the laser beam deflections via a position sensing detector (Pacific Silicon Sensors) at $20 \mathrm{kHz}$. The trap is calibrated for force measurement using the stokes drag method.

Linear viscoelastic properties are determined from thermal fluctuations of a trapped microsphere, measured by recording the associated laser deflections for 180 seconds at $20 \mathrm{kHz}$. Linear viscoelastic moduli, i.e. the 
elastic modulus $G^{\prime}(\omega)$ and the viscous modulus $G^{\prime \prime}(\omega)$, were extracted from the thermal fluctuations using the generalized Stokes-Einstein relation (GSER) as described in ref ${ }^{17}$. The procedure requires extracting the normalized mean-squared displacements $\left(\pi(\tau)=\left\langle r^{2}(\tau)\right\rangle / 2\left\langle r^{2}\right\rangle\right.$ ) of the thermal forces, averaged over all trials, which is then converted into the Fourier domain via:

$-\omega^{2} \pi(\omega)=\left(1-e^{-i \omega \tau_{1}}\right) \frac{\pi\left(\tau_{1}\right)}{\tau_{1}}+\dot{\pi}_{\infty} e^{-i \omega t_{N}}+\sum_{k=2}^{N}\left(\frac{\pi_{k}-\pi_{k-1}}{\tau_{k}-\tau_{k-1}}\right)\left(e^{-i \omega \tau_{k-1}}-e^{-i \omega \tau_{k}}\right)$,

where $\tau, 1$ and $\mathrm{N}$ represent the lag time and the first and last point of the oversampled $\pi(\tau) . \dot{\pi}_{\infty}$ is the extrapolated slope of $\pi(\tau)$ at infinity. Oversampling is done using the MATLAB function PCHIP. $\pi(\omega)$ is related to viscoelastic moduli via:

$G^{*}(\omega)=G^{\prime}(\omega)+i G^{\prime \prime}(\omega)=\left(\frac{k}{6 \pi R}\right)\left(\frac{1}{i \omega \pi(\omega)}-1\right)$,

where $R$ and $k$ represent the radius of the microsphere and trap stiffness. We computed the complex viscosity $\eta^{*}(\omega)$ via $\eta^{*}(\omega)=\left[\left(G^{\prime}(\omega)\right)^{2}+\left(G^{\prime \prime}(\omega)\right)^{2}\right]^{1 / 2} / \omega$.

Nonlinear microrheology measurements are performed by displacing a trapped microsphere, $x=30 \mu \mathrm{m}$, through the sample at speeds of $v=2.5-120 \mu \mathrm{m} / \mathrm{s}$ using a piezoelectric nanopositioning stage (Mad City Laboratories) to move the sample relative to the microsphere. We convert the distance to strain via $\gamma=$ $x / 2 R$, and convert speeds to strain rates via $\dot{\gamma}=3 v / \sqrt{2} R\left(2.4-113 \mathrm{~s}^{-1}\right){ }^{18}$. The strain of 6.7 is much higher than the critical value of 1 for nonlinearity and our chosen strain rates are higher than the terminal relaxation frequencies $\omega_{T}=\lim _{\omega \rightarrow 0} \omega G^{\prime \prime} / G^{\prime}$ of all DNA-MT composites under investigation. For both linear and nonlinear measurements, all data is recorded at $20 \mathrm{kHz}$, and at least 15 trials are conducted, each with a new microsphere in an unperturbed location. Presented data is an average of all trials.

Confocal microscopy: Microtubules in DNA-MT composite networks are imaged using a Nikon A1R laser scanning confocal microscope with a $60 \times 1.4$ NA objective. $10 \%$ of tubulin dimers comprising microtubules are rhodamine-labeled to enable imaging using a $561 \mathrm{~nm}$ laser with $561 \mathrm{~nm}$ excitation and $595 \mathrm{~nm}$ emission filters. DNA in composites is unlabeled. $512 \times 512$ pixel images $(212 \mu \mathrm{m} \times 212 \mu \mathrm{m})$ are taken at 20 different locations in the sample for each DNA-MT composite. Spatial image autocorrelation analysis is performed on each image using a custom-written python script ${ }^{19,20}$. Autocorrelation curves, $g(r)$, for each image are obtained by measuring the correlation in intensity $I(r)$ as a function of distance $r .{ }^{19}$ Specifically, $g(r)$ is computed from $I(r)$ via:

$$
g(r)=\frac{F^{-1}\left(|F(I(r))|^{2}\right)}{[I(r)]^{2}}
$$

where $F$ and $F^{-1}$ represent fast Fourier and inverse Fourier transforms. The correlation length $\xi$ is obtained by fitting autocorrelation curves to $g(r)=A e^{-r / \xi}$. 

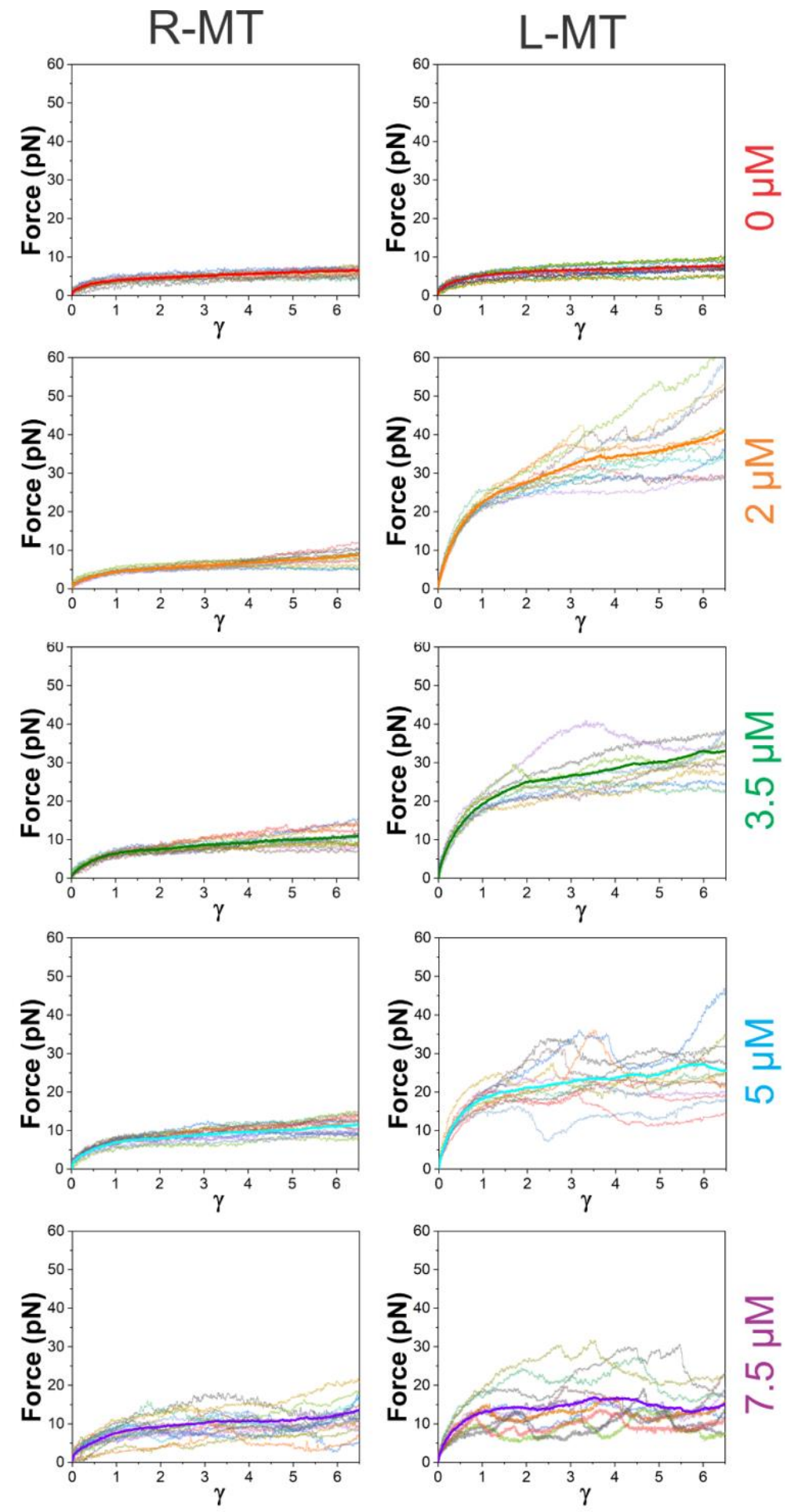

Figure S1. Measured forces in response to strain of rate $\dot{\gamma}=9.4 \mathrm{~s}^{-1}$ for DNA-MT composites. The individual trials for R-MT (left column) and L-MT (right column) composites with varying tubulin concentrations (listed to the right of each row) are shown with 50\% transparency, whereas the averages of all trials are shown in bold, thick lines. The color scheme of the average curves is identical to Fig 3 in the main text. Note the spread in the individual trials as well as the 'bumpiness' of individual curves increases with increasing tubulin concentration. 

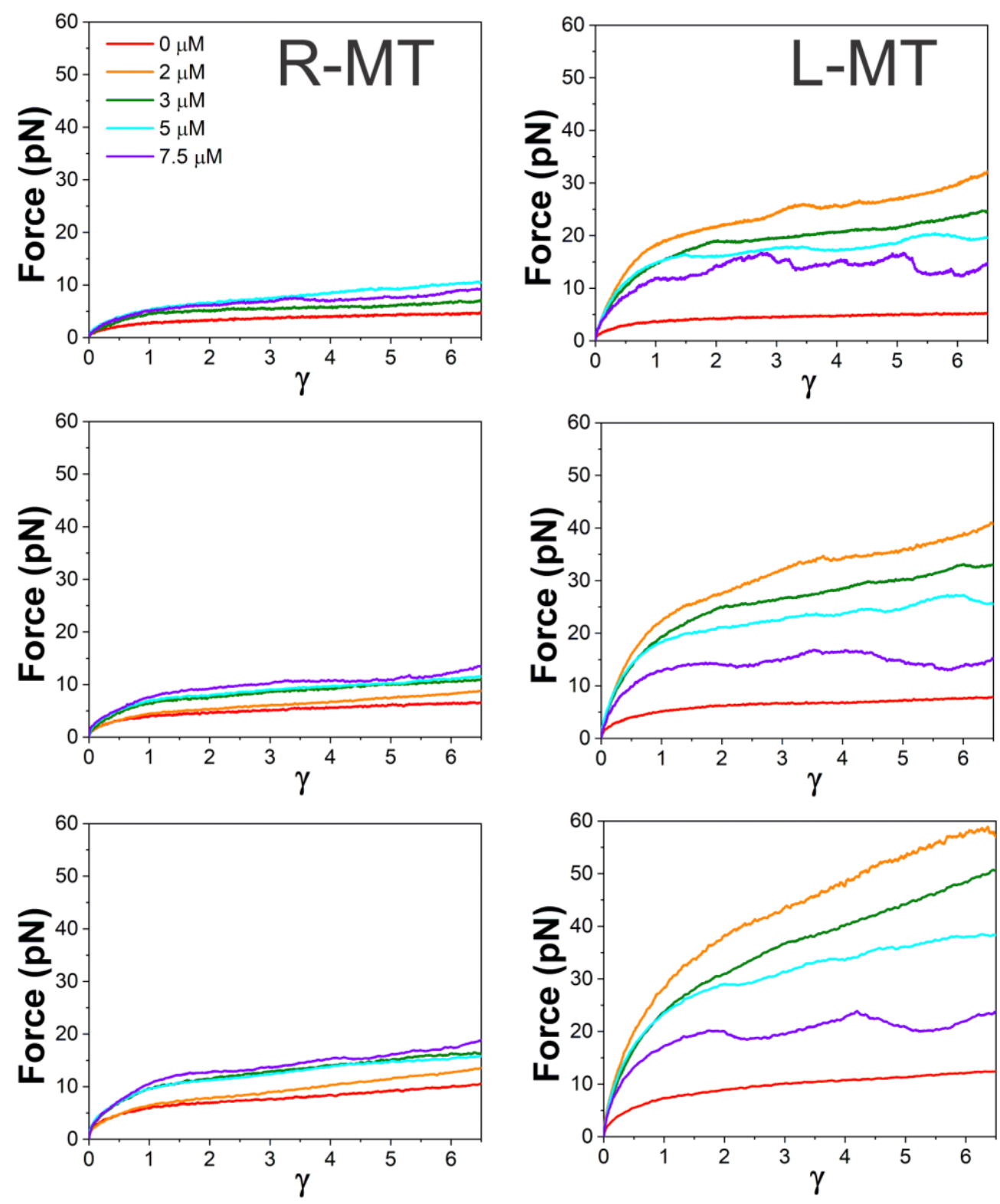

Figure S2. The nonlinear force responses of DNA-MT composites for different strain rates. Measured force in response to strain of rates $\dot{\gamma}=4.7 \mathrm{~s}^{-1}$ (top row), $9.4 \mathrm{~s}^{-1}$ (middle row) and $19 \mathrm{~s}^{-1}$ (bottom row) for composites with ring (left column) or linear (right column) DNA. The color scheme for the varying tubulin concentrations is shown in the legend in the top left graph. 

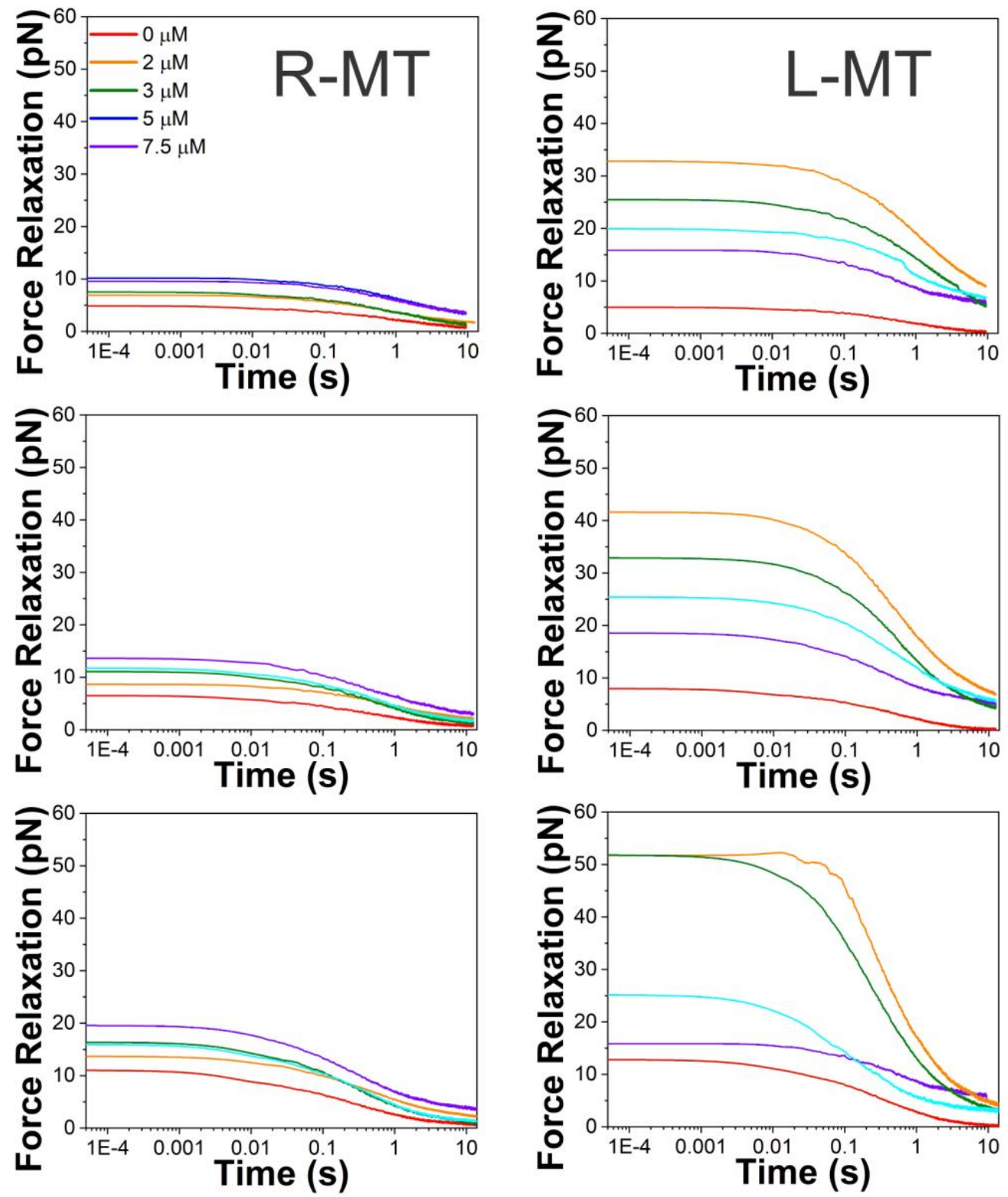

Figure S3. The force relaxation behavior of DNA-MT composites for different strain rates. Measured force relaxations in response to strain of rates $\dot{\gamma}=4.7 \mathrm{~s}^{-1}$ (top row), $9.4 \mathrm{~s}^{-1}$ (middle row) and $19 \mathrm{~s}^{-1}$ (bottom row) for composites with ring (left column) or linear (right column) DNA. The color scheme for the varying tubulin concentrations is shown in the legend of the top left graph. 

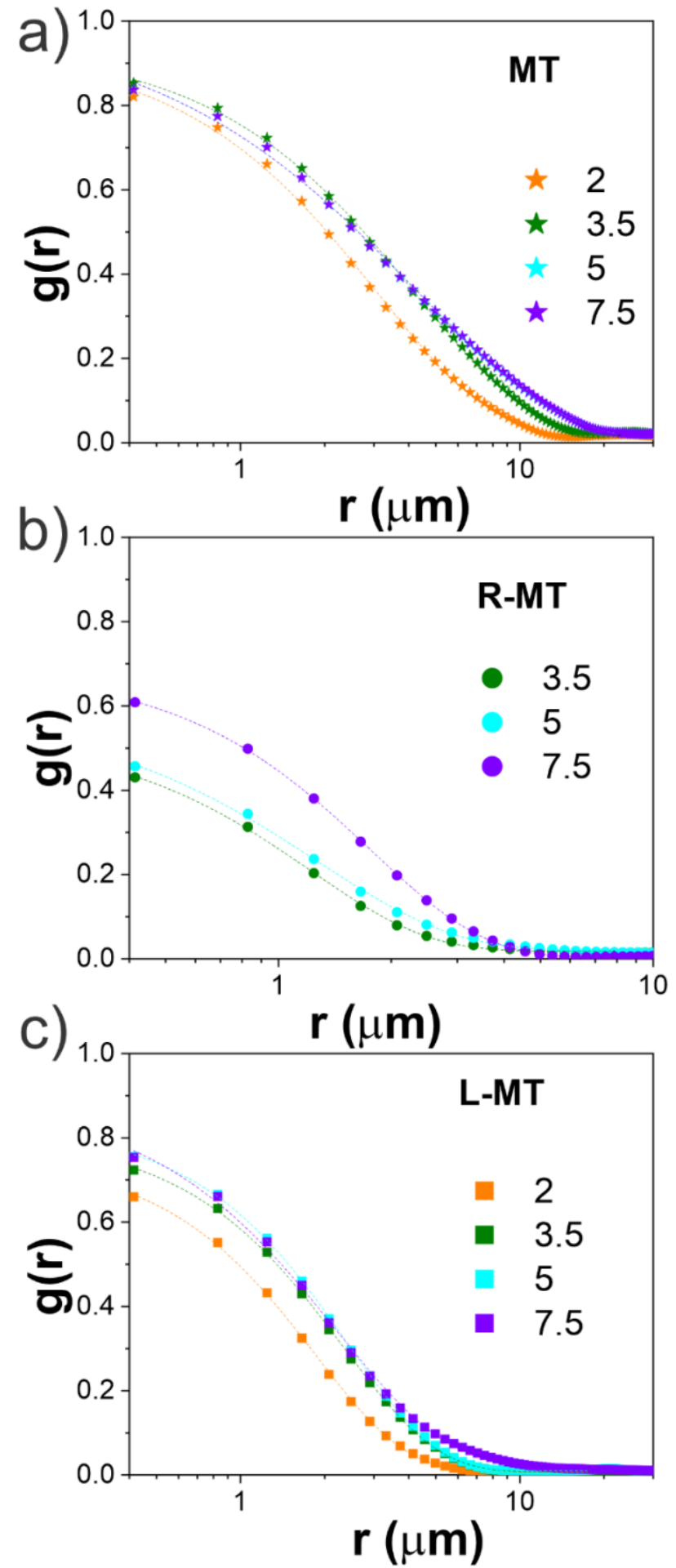

Figure S4. Average spatial image autocorrelation curves of DNA-MT composites. Each curve is an average of $g(r)$ curves computed for 20 different images of rhodamine-labeled microtubules taken at different locations of 2 independent samples for (a) microtubules without DNA, (b) R-MT composites and (c) L-MT composites with varying tubulin concentrations shown in $\mu \mathrm{M}$ in the legends. 


\section{Section 2: Theory for phase behavior of a composite of stiff and flexible polymers}

The semi-grand potential for a solution of hard rod-like colloids and flexible polymers is

$$
F=F_{R}\left(N_{R}, V,[f]\right)-\Pi\left(\mu_{P}\right) V_{f}
$$

Where $F_{R}$ is the Helmoltz free energy of a system of $N_{R}$ rods in a volume $V$ and $\Pi=\mu_{p} n_{p}$ is the osmotic pressure exerted by a reservoir of polymer molecules, equal to the chemical potential multiplied by the number density of polymer molecules. Finally, $V_{f}$ is the free volume available to the polymer computed by the average volume available to polymers and $f$ the orientation distribution of the rods. This function captures the fact that the rods can be in an isotropic or nematic state and assumes a shape that minimizes the free energy.

Free energy of rod colloids

To express $F_{R}$ as a function of the density including corrections beyond the second virial coefficient we use the Scaled Particle Theory (SPT) description ${ }^{21,22}$ :

$$
\frac{F_{R}}{N_{c} k_{B} T}=\log n_{c}-\log (1-\phi)+\sigma+X_{2} \frac{\phi}{1-\phi}+\frac{1}{2} X_{3}\left(\frac{\phi}{1-\phi}\right)^{2}
$$

Where $n_{c}=N_{c} / V$ is the colloid particle density,

$$
\phi=n_{c}\left(\frac{\pi}{6} D^{3}+\frac{\pi}{4} D^{2} L\right)
$$

their volume fraction. The coefficients $X_{2}$ and $X_{3}$ are given by

$$
\begin{gathered}
X_{2}=3+\frac{3(\gamma-1)^{2}}{3 \gamma-1} \rho[f] \\
X_{3}=\frac{12 \gamma(2 \gamma-1)}{(3 \gamma-1)^{2}}+\frac{12 \gamma(\gamma-1)^{2}}{(3 \gamma-1)^{2}} \rho[f]
\end{gathered}
$$

Where $\gamma=(L+D) / D$ and the quantities

$$
\begin{gathered}
\sigma=\int d \Omega f \log (4 \pi f) \\
\rho=\frac{4}{\pi} \iint d \Omega \mathrm{d} \Omega^{\prime} f f^{\prime} \sin (\theta)
\end{gathered}
$$

And $\theta$ is the angle between two rod-like colloids. To simplify the calculations, we will use the ansatz that $f$ is a Gaussian distribution - this reduces the problem of finding the free energy minimizing orientation distribution of colloids to that of minimizing a variational parameter $\alpha$ defined via the function $f(\theta)=$ $\frac{\alpha}{4 \pi} e^{-\frac{1}{2} \alpha \theta^{2}}$ for $0<\theta<\frac{\pi}{2}$ and $f(\theta)=\frac{\alpha}{4 \pi} e^{-\frac{1}{2} \alpha(\pi-\theta)^{2}}$ for $\frac{\pi}{2}<\theta<\pi$. Importantly, using large $\alpha$ (nematic phase), one finds 


$$
\sigma=\sigma(\alpha) \simeq \log (\alpha)-1
$$

and

$$
\rho=\rho(\alpha) \simeq 4 / \sqrt{\pi \alpha}
$$

In the isotropic phase, these terms are $\sigma=0$ and $\rho=1$.

Free volume of polymers

To obtain the free volume available to polymers, one can employ Widom's particle insertion method, which relates the chemical potential of a polymer species to the work needed to insert one such a polymer in the system. We thus write

$$
\mu_{p}=k_{B} T \log \frac{N_{p}}{V_{f}}=k_{B} T\left(\log \frac{N_{p}}{V}-\log \frac{V_{f}}{V}\right)
$$

Which also equals

$$
\mu_{p}=k_{B} T \log \frac{N_{p}}{V}+W
$$

where $\mathrm{W}$ is the work to insert a polymer molecule in the system.

Putting the two equations together one obtains

$$
v=\frac{V_{f}}{V}=e^{-W / k_{B} T}=(1-\phi) \exp \left(-A y-B y^{2}-C y^{3}\right)
$$

Where $\mathrm{y}=\phi /(1-\phi)$ and

$$
\begin{gathered}
A=\frac{6 \gamma}{3 \gamma-1} q+\frac{3(\gamma+1)}{3 \gamma-1} q^{2}+\frac{2}{3 \gamma-1} q^{3} \\
B=\frac{1}{2}\left(\frac{6 \gamma}{3 \gamma-1}\right)^{2} q^{2}+\left(\frac{6}{3 \gamma-1}+\frac{6(\gamma-1)^{2}}{3 \gamma-1} \rho[f]\right) q^{3} \\
C=\frac{2}{3 \gamma-1}\left(\frac{12 \gamma(2 \gamma-1)}{(3 \gamma-1)^{2}}+\frac{12 \gamma(\gamma-1)^{2}}{(3 \gamma-1)^{2}} \rho[f]\right) q^{3}
\end{gathered}
$$

With $q=\sigma / D$ is the ratio between the diameter of the flexible polymer and the diameter of the rod-like colloid and $\gamma=(L+D) / D^{21}$. These quantities are the most important to applying this model to our work. Specifically, $\sigma$ is different for linear and ring polymers, and, in the case of DNA, is $\sim 1.58 \mathrm{x}$ smaller for the latter. Based on previous measurements, we use $\sigma_{L} \simeq 2 R_{G, L} \simeq 1.76 \mu \mathrm{m}$ for linear DNA chains and $\sigma_{R} \simeq$ $2 R_{G, R} \simeq 1.04 \mu \mathrm{m}$ for the rings ${ }^{1}$. The diameter and length of the rod-like colloid (the microtubules) are kept 
fixed at $D=1 \mu \mathrm{m}$ and $L=10 \mu \mathrm{m}$, respectively. While $L$ is based on experimental observations, $D$ is larger than the expected MT diameter of $\sim 25 \mathrm{~nm}$, due to the numerical instability of the theory for smaller values of $D$. Further, negatively charged microtubules have a screening length, dependent on the ionic conditions of the solution, that increases their 'effective' diameter, so $25 \mathrm{~nm}$ is an underestimate. Nevertheless, to address this discrepancy we evaluate the phase diagram in terms of MT number concentration rather than volume fraction (as shown in Fig 6).

Combining Eqs. (3-5) into (1) yields a semi-grand canonical free energy with one $(\alpha)$ variational parameter that needs to be tuned to its free-energy-minimising value. To find it, we numerically compute the minimum of Eq. (1) as a function of $\alpha$ and for different values of $n_{c}$ (or equivalently $\phi$ ) and we store its root $\alpha_{m}(\phi)$. This parameter is found to undergo a jump from 0 to $>0$ at a critical value $\phi_{c}$ that signals the onset of the nematic transition. To find the binodals we then solve the standard coexistence equations, i.e.

$$
\begin{aligned}
& \mu_{c}\left(\phi_{I}, f_{I}\right)=\mu_{c}\left(\phi_{N}, f_{N}\right) \\
& \Pi_{c}\left(\phi_{I}, f_{I}\right)=\Pi_{c}\left(\phi_{N}, f_{N}\right)
\end{aligned}
$$

Where $\mu_{c}=\frac{d F^{*}}{d N_{c}}=\frac{d\left(F_{R}^{*} / V\right)}{d n_{c}}-\Pi_{p} \frac{d v}{d n_{c}}$ is the total chemical potential (evaluated from $F^{*}=F\left(\phi, \alpha=\alpha_{m}\right)$ ) and $\Pi_{c}=-\frac{d F^{*}}{d V}=\Pi_{0}+\Pi_{p}\left(v-n_{c} \frac{d v}{d n_{c}}\right)$ is the pressure (to compute these we make use of $\frac{N_{c}}{V}=n_{c}=$ $\left.\phi /\left(\frac{\pi}{6} D^{3}+\frac{\pi}{4} D^{2} L\right)\right)$. The coexistence equations essentially encode the fact that, in equilibrium, both chemical potential and pressure must be equal in the two (isotropic and nematic) phases. Finally, the real volume fraction of polymer in the two phases is found by multiplying the polymer concentration in the "reservoir" at pressure $\Pi_{p}$ and volume fraction $\phi_{p}$ by the free volume $v(\phi)=(1-\phi) \exp \left(-A y-B y^{2}-\right.$ $\left.C y^{3}\right)$ where $y=\phi /(1-\phi)$. By doing so, we plot the phase diagram in the space of DNA and microtubule volume fractions. In the main text, we then divide the MT volume fraction by the volume of one MT to find the number concentration $n$ (see Fig 6). Converting to number concentration allows us to better compare with experiments by removing the dependence on the unknown exact volume of MTs.

Because the DNA in our system is overlapping (i.e., $c>c^{*}$ ), it is possible that the principal polymer lengthscale that dictates depletion interactions in SPT (i.e., $\sigma$ ) is the correlation blob size of the DNA $\zeta$ (see Section $\mathrm{S} 1$ ) rather than $R_{G}{ }^{23,24}$. The blob sizes for our ring and linear DNA solutions are $\zeta_{R} \cong 185 \mathrm{~nm}$ and $\zeta_{L} \cong 102 \mathrm{~nm}$, respectively, such that $\zeta_{L} \cong 1.8 \zeta_{R}$. While these lengthscales are appreciably smaller than $R_{G, R}$ and $2 R_{G, L}$, the relationship between the ring and linear lengthscales is similar. As such, the corresponding SPT phase diagram is qualitatively the same as shown in Fig 6 (see Fig S5).

Alternative theories that consider a truncated virial expansion of the free energy to explain the phase separation of rod-like colloids and flexible coils can be considered and yield qualitatively similar phase diagrams ${ }^{25}$. 


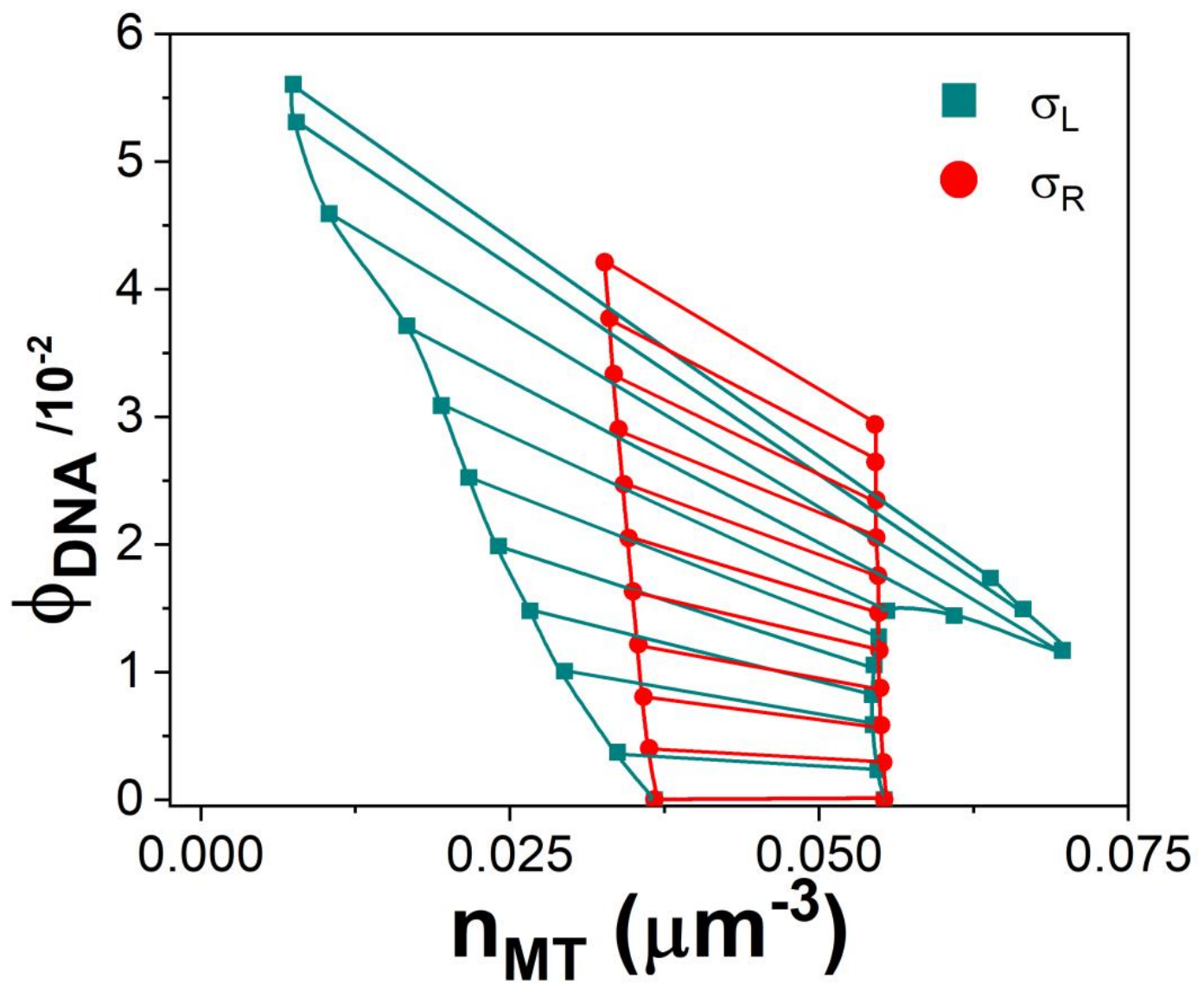

Figure S5. Results of Scaled Particle Theory using the correlation blob size $\zeta$ rather than $\boldsymbol{R}_{G}$ as the principal DNA lengthscale $\sigma$. Phase diagram showing results using SPT for a composite of rod-like particles (MTs) and flexible polymer coils (DNA) with $\sigma_{L} \simeq \zeta_{L}$ and $\sigma_{R} \simeq \zeta_{R}$, and for varying DNA volume fraction $\phi_{D N A}$ and MT number concentration $n_{M T}$. The points mark the binodal lines separating the isotropic (left) and nematic (right) phases for the microtubules in the presence of ring (red) or linear (dark cyan) DNA. The lines connecting the binodals are the corresponding tie lines. The difference between ring and linear DNA is their different sizes, $\sigma_{R}$ and $\sigma_{L}$, with rings being smaller than linear DNA. The coexistence regions between the binodals, indicate the region in which isotropic and nematic MT phases coexist, which manifests as MT flocculation in experiments. Note that the coexistence region is significantly smaller for ring DNA and shifted towards higher $n_{M T}$ values, as seen in experiments. 


\section{References}

1. Robertson, R. M.; Laib, S.; Smith, D. E., Diffusion of isolated DNA molecules: Dependence on length and topology. Proceedings of the National Academy of Sciences 2006, 103 (19), 7310-7314.

2. Laib, S.; Robertson, R. M.; Smith, D. E., Preparation and Characterization of a Set of Linear DNA Molecules for Polymer Physics and Rheology Studies. Macromolecules 2006, 39 (12), 4115-4119.

3. Peddireddy, K. R.; Lee, M.; Schroeder, C. M.; Robertson-Anderson, R. M., Viscoelastic properties of ring-linear DNA blends exhibit nonmonotonic dependence on blend composition. Physical Review Research 2020, 2 (2), 023213.

4. Parisi, D.; Ahn, J.; Chang, T.; Vlassopoulos, D.; Rubinstein, M., Stress Relaxation in Symmetric Ring-Linear Polymer Blends at Low Ring Fractions. Macromolecules 2020, 53 (5), 1685-1693.

5. Robertson, R. M.; Smith, D. E., Self-Diffusion of Entangled Linear and Circular DNA Molecules: Dependence on Length and Concentration. Macromolecules 2007, 40 (9), 3373-3377.

6. Doi, M.; Edwards, S. F., The Theory of Polymer Dynamics Oxford University Press: New York, 1986; Vol. 73.

7. Robertson, R. M.; Smith, D. E., Direct Measurement of the Intermolecular Forces Confining a Single Molecule in an Entangled Polymer Solution. Physical Review Letters 2007, 99 (12), 126001.

8. Tubulin Protein (Rhodamine Labeled, from porcine brain). https://www.cytoskeleton.com/pdfstorage/datasheets/t1590m.pdf.

9. Ricketts, S. N.; Francis, M. L.; Farhadi, L.; Rust, M. J.; Das, M.; Ross, J. L.; RobertsonAnderson, R. M., Varying crosslinking motifs drive the mesoscale mechanics of actin-microtubule composites. Scientific Reports 2019, 9 (1), 12831.

10. Wen, Q.; Janmey, P. A., Polymer physics of the cytoskeleton. Current Opinion in Solid State and Materials Science 2011, 15 (5), 177-182.

11. Lin, Y.-C.; Koenderink, G. H.; MacKintosh, F. C.; Weitz, D. A., Viscoelastic Properties of Microtubule Networks. Macromolecules 2007, 40 (21), 7714-7720.

12. Gittes, F.; Mickey, B.; Nettleton, J.; Howard, J., Flexural rigidity of microtubules and actin filaments measured from thermal fluctuations in shape. Journal of Cell Biology 1993, 120 (4), 923934.

13. Hawkins, T.; Mirigian, M.; Selcuk Yasar, M.; Ross, J. L., Mechanics of microtubules. Journal of Biomechanics 2010, 43 (1), 23-30.

14. Ying, Q.; Chu, B., Overlap concentration of macromolecules in solution. Macromolecules 1987, 20 (2), 362-366.

15. Falzone, T. T.; Blair, S.; Robertson-Anderson, R. M., Entangled F-actin displays a unique crossover to microscale nonlinearity dominated by entanglement segment dynamics. Soft Matter 2015, 11 (22), 4418-4423.

16. Gurmessa, B.; Francis, M.; Rust, M. J.; Das, M.; Ross, J. L.; Robertson-Anderson, R. M., Counterion crossbridges enable robust multiscale elasticity in actin networks. Physical Review Research 2019, 1 (1), 013016.

17. Tassieri, M.; Evans, R. M. L.; Warren, R. L.; Bailey, N. J.; Cooper, J. M., Microrheology with optical tweezers: data analysis. New Journal of Physics 2012, 14 (11), 115032.

18. Squires, T. M., Nonlinear Microrheology: Bulk Stresses versus Direct Interactions. Langmuir 2008, 24 (4), 1147-1159.

19. Robertson, C.; George, S. C., Theory and practical recommendations for autocorrelation-based image correlation spectroscopy. Journal of biomedical optics 2012, 17 (8), 080801.

20. Lee, G.; Leech, G.; Lwin, P.; Michel, J.; Currie, C.; Rust, M. J.; Ross, J. L.; McGorty, R. J.; Das, M.; Robertson-Anderson, R. M., Active Cytoskeletal Composites Display Emergent Tunable Contractility and Restructuring. arXiv preprint arXiv:2104.04113 2021.

21. Lekkerkerker, H. N. W.; Stroobants, A., Phase behaviour of rod-like colloid+flexible polymer mixtures. Il Nuovo Cimento D 1994, 16 (8), 949. 
22. Warren, P. B., Depletion effect in a model lyotropic liquid crystal-theory. Journal de Physique I 1994, 4 (2), 237-244.

23. Verma, R.; Crocker, J. C.; Lubensky, T. C.; Yodh, A. G., Entropic Colloidal Interactions in Concentrated DNA Solutions. Physical Review Letters 1998, 81 (18), 4004-4007.

24. Verma, R.; Crocker, J. C.; Lubensky, T. C.; Yodh, A. G., Attractions between Hard Colloidal Spheres in Semiflexible Polymer Solutions. Macromolecules 2000, 33 (1), 177-186.

25. Sear, R. P.; Jackson, G., Theory for the phase behavior of a mixture of a rodlike colloid and a rodlike polymer. The Journal of Chemical Physics 1995, 103 (19), 8684-8693. 